\title{
Does telomerase protein protect our neurons?
}

\author{
Gabriele C Saretzki
}

Institute for Cell and Molecular Biosciences, Newcastle Institute for Ageing, Campus for Ageing and Vitality, Newcastle University, UK

\section{Article Info}

\section{Article Notes}

Received: 05/05/2016

Accepted: 05/18/2016

\section{*Correspondence:}

\section{Dr. Gabriele C Saretzki}

Institute for Cell and Molecular Biosciences, Newcastle

Institute for Ageing, Campus for Ageing and Vitality, Newcastle

University,

Newcastle upon Tyne, NE4 5PL, UK

Telephone: +44 1912081214

Fax: +44 1912081101

Email: gabriele.saretzki@ncl.ac.uk

C 2016 G Saretzki. This article is distributed under the terms of the Creative Commons Attribution 4.0 International License

\section{Keywords}

Telomerase

Brain

Neuron

Mitochondria

Neurodegeneration

Protection

\begin{abstract}
Telomerase is best known for its canonical function in telomere maintenance. However, a growing number of non-telomeric functions have been described. Several groups have found the telomerase protein TERT to persist in adult brain neurons. A protective role for the telomerase protein TERT had been demonstrated in cultivated mouse neurons during brain development, against excitotoxic stresses from N-methyl-D-aspartate (NMDA) and glutamate and agents known to be involved in neurodegenerative diseases such as beta amyloid peptides and hyperphosphorylated tau. In contrast, lack of telomerase and TERT protein increase oxidative stress and decrease neuronal survival. Research on telomerase and TERT protein in human neurodegenerative diseases is a relatively new field. However, there is emerging evidence of a beneficial role of telomerase in human brains and animal models of neurodegenerative diseases that suggests to explore the possibility of using telomerase activators as neuroprotective agents to combat brain ageing and to ameliorate neurodegenerative diseases such as Parkinson's and Alzheimer's diseases. The current mini-review summarises the knowledge about this developing novel area of brain research.
\end{abstract}


Telomerase is a reverse transcriptase best known as the enzyme that extends and maintains telomeres, the protective ends of linear chromosomes, in dividing cells. It consists of the catalytic protein TERT (Telomerase Reverse Transcriptase) and an RNA subunit which contains the template region for telomere synthesis. However, in most human somatic cells telomerase is downregulated early during development ${ }^{1,2}$ while some cell types such as endothelial cells, lymphocytes and adult stem cells express some activity throughout life. Due to its own RNA moiety, telomerase is able to extend the 3 ' overhang of telomeres, generated during DNA replication and thus counteract telomere shortening. Without sufficient telomerase activity present, telomeres shorten due to the inability of conventional DNA polymerases to replicate the lagging strand at the end of chromosomes, the so called "end replication problem"3,4. Importantly, also oxidative stress has been shown to be a major reason for telomere shortening ${ }^{5}$. However, telomere shortening happens mainly in dividing cells and this division is required for translating accumulated DNA damage and DNA breaks into telomere shortening ${ }^{6}$. In addition, telomeres are not well repaired ${ }^{7}$. DNA damage however, can also happen in non-dividing cells such as neurons and it was even shown that the grade of maturation might play a role with newly formed neurons possibly more sensitive against DNA damage than mature ones ${ }^{8}$. Whether and to what extent all neuron types and brain areas are prone to DNA damage remains a research question still under debate ${ }^{9}$. In some neuron types such as Purkinje neurons, both DNA damage and cellular senescence as well as telomerase have been detected $^{10-12}$.

The research on the involvement of telomerase in neurons and the brain was pioneered by Mark Mattson's group more than 15 years ago. They showed that cultivated neurons from mouse embryos need telomerase activity for protection and development and that telomerase promotes their survival against trophic factor withdrawal, glutamateinduced excitotoxicity and amyloid peptides in cultured mouse primary neurons $\mathrm{s}^{13-16}$. Analysing telomerase activity as well as the expression of its protein part TERT it became apparent that, while telomerase activity is downregulated early during brain development in humans ${ }^{1}$ and postnatally in rodents, the TERT protein seemed to persist even in adult mouse brains ${ }^{17,18}$.

In addition to telomere-dependent, canonical functions of telomerase, more and more telomere-independent, non-canonical functions of the TERT protein became apparent over the last decade (for reviews $s^{19,20}$ ). This includes subcellular shuttling of TERT from the nucleus to mitochondria upon increased oxidative stress ${ }^{21-24}$. In addition to a nuclear localisation signal (NLS) and a nuclear exclusion signal (NES) ${ }^{25,26}$ TERT in higher organisms such as mammals, also contains a specific mitochondrial localisation signal (MLS) ${ }^{21}$. The nuclear exclusion upon oxidative stress is dependent on Src kinase and Ran GTPase $^{27}$. TERT within mitochondria exerts a protective function with a decrease of mitochondrial and intracellular oxidative stress, decrease of apoptosis and protection of mitochondrial and nuclear $\mathrm{DNA}^{22-24}$ although the exact underlying mechanisms still remain elusive. Binding to mitochondrial DNA, improvement of mitochondrial respiration and changes in cellular antioxidants have been suggested ${ }^{23,28,29}$. Interestingly, Santos' group demonstrated that the TERT protein can exert its reverse transcriptase function in mitochondria by using mitochondrial tRNAs as templates for reverse transcription ${ }^{29}$ although the biological significance of that novel catalytic function of telomerase within mitochondria has not been demonstrated yet. There is an ongoing debate in the telomerase field whether some non-canonical functions are dependent on the presence of the catalytic function of TERT while others are not (for review $\mathrm{see}^{20}$ ). Demonstrating the catalytic function of TERT within mitochondria does not answer this question since no direct correlation between the catalytic activity of TERT in mitochondria and its protective function has been confirmed. It is also not known whether catalytic activity of TERT is essential or required for the protection of neurons by TERT. The protective role of mammalian TERT in mitochondria has recently been demonstrated even in yeast where it increased the resistance against oxidative stress without interacting with telomeres or the yeast TERT which lacks the mitochondrial localisation signal ${ }^{30}$.

Our group also found that upon treatment with hydrogen peroxide or irradiation cancer cells exclude TERT protein in a heterogeneous manner and intriguingly, nuclear DNA damage correlated positively with TERT protein remaining within the nucleus; while cells with cytoplasmic/mitochondrial TERT localisation had lower nuclear DNA damage, lower levels of reactive oxygen species (ROS) and hardly any apoptosis. In contrast, cells with a nuclear TERT localisation had high amounts of DNA damage, oxidative stress as well as apoptosis ${ }^{24}$. This finding corresponds well with results from experiments that used a hTERT protein with a mutant NES rendering the protein unable to leave the nucleus resulting in increased nuclear and mitochondrial DNA damage as well as higher levels of oxidative stress ${ }^{32,33}$.

There are various mechanisms of neuronal cell death in the brain during neurodegeneration. While in Parkinson's disease (PD) apoptosis might occur as the pre-dominant mechanism, in Alzheimer's disease (AD) the exact mechanism of neuronal degeneration is still not known ${ }^{31}$. Consequently, the involvement of TERT into neuronal apoptosis has not been investigated yet.

It has not been analysed in detail whether nuclear 
localisation of TERT is the default situation in all cell types. Immune cells such as lymphocytes have their TERT protein in the cytoplasm under unstimulated conditions and transport it into the nucleus upon antigen or inflammatory stimulation (see Figure $1 \mathrm{~A}$ ) and activate telomerase activity and nuclear translocation by phosphorylating TERT on serine 227 by $\mathrm{AKT}^{34-36}$. In contrast, it is thought that TERT and telomerase activity are localised within the nucleus under non-stressed conditions ${ }^{24}$ (see Figure 1B).

Intriguingly, Iannilli et al showed that in fully differentiated neurons the majority of cytoplasmic TERT forms a complex with RNA granules and binds to the mRNA of p $15^{\mathrm{INK} 4 \mathrm{~b}}$, a pro-survival cell cycle inhibitor ${ }^{37}$. Upon cellular stress TERT and p15 dissociated resulting in translation of p15 while TERT was free to shuttle to mitochondria. The authors also showed that the anti-apoptotic functions of TERT and p15 in cultivated rat hippocampal neurons were mutually dependent on each other ${ }^{37}$. In general, Iannilli's findings suggested that TERT protein is not necessarily localised in the nucleus of neurons corresponding to reports from Spilsbury et al.$^{38}$ who also found TERT signals predominantly outside the nucleus in hippocampal human and cultivated mouse neurons although contradicting results exist and changes might occur with the age of the $\operatorname{organism}^{10}$.

Mattson's group also showed that presence of telomerase is able to protect against apoptosis, withdrawal of growth factors and glutamate treatment (excitotoxicity) as well as treatment with amyloid precursor protein in cultivated primary mouse neurons ${ }^{13,16}$. Thus, a potential connection between the presence of TERT protein in adult brains and neurodegeneration was possible ${ }^{39}$. Recently, Esther Priels' group has demonstrated that boosting telomerase levels in mice by using specific compounds that activate telomerase, can ameliorate NMDA toxicity in wild type mice ${ }^{40}$. This seems to correspond well to a previous study in mouse brains in vivo that showed that overexpressing TERT in neurons did not result in any telomerase activity but improved protection of neurons from NMDA excitotoxicity as well as mitochondrial functions such as mitochondrial membrane potential ${ }^{41}$. Importantly, Eitan and co-workers also showed that compounds that activate telomerase can ameliorate symptoms and disease severity in a mouse model of Amyotrophic Lateral Sclerosis (ALS), again suggesting a beneficial effect of telomerase/TERT levels in the brain of adult mice of a neurodegenerative mouse model ${ }^{40}$.

It had been demonstrated that telomerase activity is downregulated very early duringhuman brain development ${ }^{1}$. However, not much research had been performed on adult human brains. Our group used human brain tissue from the Newcastle Brain Tissue Resource (NBTR) and analysed the hippocampus of donors with different Braak stages of Alzheimer's disease (AD) and compared them to agematched healthy controls. We found that only neurons, but not astrocytes expressed any TERT protein while there was some expression in human microglia, presumably due to their nature being macrophages ${ }^{38}$. While our initial hypothesis was that TERT protein might decrease due to disease progression, we could not find any evidence of that using different techniques such as Western blotting
A

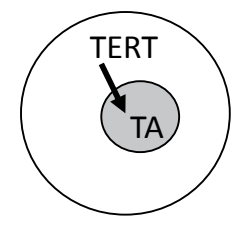

B

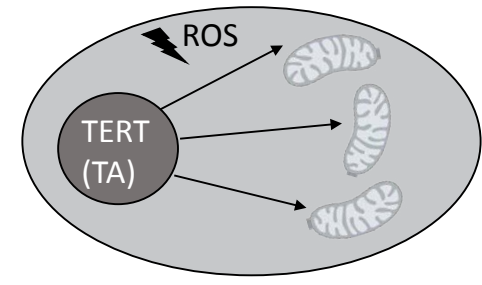

C

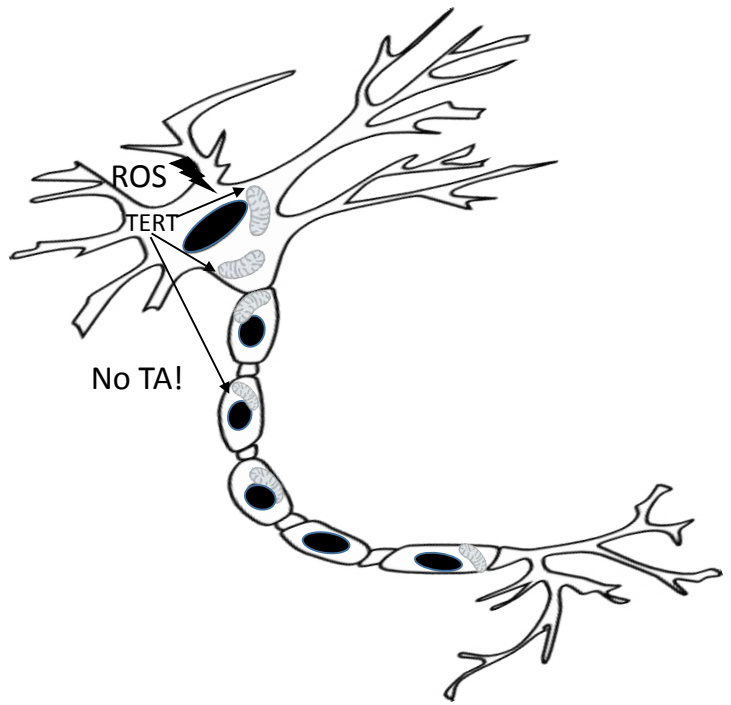

Figure 1: Subcellular localisation of TERT protein as well as the presence or absence of telomerase activity (TA) in different cell types. A: Lymphocytes: TERT enters nucleus upon stimulation, the role of ROS for changes in TERT localisation and TA are not known. B: Cancer cells: TERT localises in the nucleus as default, but can shuttle to mitochondria upon oxidative stress (ROS). C: Adult neurons: TERT is localised in the cytoplasm and might form complex with other molecules that is interrupted upon oxidative stress ${ }^{37}$ and localises within mitochondria. ${ }^{38,11}$ 
and immuno-fluorescence staining for TERT protein ${ }^{38}$. However, we found that there was a significant increase in the amount of mitochondrial TERT localisation in hippocampal areas CA1-3 of advanced AD cases (Braak stage 6) compared to healthy controls (Braak stage 0). Oxidative stress and mitochondrial dysfunction increase during brain ageing and are suggested to be involved in the pathogenesis of Alzheimer's disease (AD) and other neurodegenerative diseases. In both $\mathrm{AD}$ and animal models of neurodegenerative diseases, chronically increased oxidative stress levels have been found to represent one of the earliest changes before specific pathologies such as amyloid- $\beta$ (A $\beta)$ plaques and neurofibrillary tangles occur ${ }^{42,43}$. Our findings together with that of Iannilli et al. ${ }^{37}$ suggest that TERT protein enters neuronal mitochondria from the cytoplasm due to increased oxidative stress where it might exert a protective function (see Figure 1C). This suggestion is also supported by our results of a lack of co-localisation of hyperphosphorylated tau protein (either as neuropil threads or neurofibrillary tangles) and TERT protein in human hippocampi of advanced Braak stages in AD brains where we found a mutual exclusion of both proteins: neurons expressing TERT did not show any tau pathology while cells with neuropil threads or neurofibrillary tangles did not show any TERT expression ${ }^{38}$. Whether high TERT expression was really protective against hyperphosphorylated tau or tau expressing neurons did displace or downregulate TERT can only be speculated at that point. However, in order to model tau pathology in an experimental system we used cultivated mouse embryonic neurons from both wild type as well as TERT knockout mice (first generation with no effect on telomere length). Similar to human brains, we found that only neurons, but not astrocytes expressed any TERT protein. Measuring oxidative stress we found that neurons lacking the TERT protein (from first generation TERT knock-out mice ${ }^{44}$ ) had higher levels of ROS after an oxidative challenge while there were no significant differences before the treatment. This finding corresponds well to that from Iannilli et al. ${ }^{37}$ and suggests that only under stressed conditions the better protection of neurons expressing TERT might become evident due to its localisation to mitochondria. Indeed, we found an increased amount of TERT protein localised to mitochondria in wild type neurons after applying oxidative stress compared to basal unstressed conditions. Transducing wild type and TERT knock-out neurons (at a time point when in wild type neurons telomerase activity was already downregulated while the TERT protein persisted) with mutated human tau protein (P301L mutation known from human tauopathies ${ }^{45}$ ), we found that neurons from TERT knock-out mice had significantly more mitochondrial ROS in dendrites, but not in the cell body. Similarly, we found more peroxidised lipids in neurons lacking TERT protein, but this time the difference was significant in the cell bodies, presumably due to a higher lipid content there ${ }^{38}$. Our data on mitochondrial localisation of TERT in neurons are in accordance with those from Eitan et al. ${ }^{11}$ who recently also demonstrated that glutamate-induced excitotoxicity stress increased the amount of TERT protein within mitochondria in mouse Purkinje neurons supporting the idea of dynamic changes in subcellular localisation of TERT due to physiological as well as pathological processes.

Other groups used TERT knock-out mice to demonstrate that lack of TERT increased infarct volumes, stroke-related neuro-inflammation, as well as enhanced behavioural abnormalities such as anxiety at higher age ${ }^{17,46}$. However, in these studies on whole brains and organisms it is not entirely clear whether it was neuronal TERT or rather TERT protein in vascular endothelial cells that contributed to the phenotype. Additional aspects of the beneficial effects of telomerase in the central nervous system have been summarised in a current review ${ }^{47}$.

Taken together, there is growing evidence for a cytoplasmic localisation of TERT protein in adult neurons that seems to correlate to a protective function in the brain that is quite distinct from telomerase enzymatic activity on telomeres. Consequently, these results suggest that an increase of TERT protein at higher age might help to prevent or ameliorate brain ageing as well as the onset and progression of various neurodegenerative diseases. Thus, telomerase might be viewed as a novel target for future strategies to combat neurodegenerative diseases. First results on mouse models of neurodegeneration are encouraging but have to be scrutinised carefully in a human setting.

\section{References}

1. Ulaner GA, Giudice LC. Developmental regulation of telomerase activity in human fetal tissues during gestation. Mol Hum Reprod. 1997; 3(9): 769-773.

2. Ulaner GA, Hu JF, Vu TH, Giudice LC, Hoffman AR. Telomerase activity in human development is regulated by human telomerase reverse transcriptase (hTERT) transcription and by alternate splicing of hTERT transcripts. Cancer Res. 1998; 58(18): 4168-4172.

3. Watson JD. The regulation of DNA synthesis in eukaryotes. Adv Cell Biol. 1971; 2: 1-46.

4. Olovnikov AM. Principle of marginotomy in template synthesis of polynucleotides. Dokl Akad Nauk SSSR. 1971; 201(6): 1496-9.

5. von Zglinicki T, Saretzki G, Docke W, Lotze C. Mild hyperoxia shortens telomeres and inhibits proliferation of fibroblasts: a model for senescence? Exp Cell Res. 1995; 220(1): 186-93.

6. Sitte N, Saretzki G, von Zglinicki T. Accelerated telomere shortening in fibroblasts after extended periods of confluency. Free Radic Biol Med. 1998; 24(6): 885-93.

7. Petersen S, Saretzki G, von Zglinicki T. Preferential accumulation of single-stranded regions in telomeres of human fibroblasts. Exp Cell Res. 1998; 239(1): 152-60.

8. Cheng A, Shin-ya K, Wan R, Tang SC, Miura T, Tang H, et al. Telomere protection mechanisms change during neurogenesis and neuronal maturation: newly generated neurons are hypersensitive to telomere and DNA damage. J Neurosci. 2007; 27(14): 3722-33. 
9. Coppedè F, Migliore L. DNA damage in neurodegenerative diseases. Mutat Res. 2015; 776: 84-97.

10. Eitan E, Tichon A, Daniel G, Priel E. Telomerase expression in adult and old mouse Purkinje neurons. Rejuvenation Research. 2012; 15: 206209.

11. Eitan E, Braverman C, Tichon A, Gitler D, Hutchison ER, Mattson MP, et al.. Excitotoxic and Radiation Stress Increase TERT Levels in the Mitochondria and Cytosol of Cerebellar Purkinje Neurons. Cerebellum. 2015.

12. Jurk D, Wang C, Miwa S, Maddick M, Korolchuk V, Tsolou A, et al. Postmitotic neurons develop a p21-dependent senescence-like phenotype driven by a DNA damage response. Aging Cell. 2012; 11(6): 996-1004.

13. Fu W, Killen M, Culmsee C, Dhar S, Pandita T, Mattson M. The catalytic subunit of telomerase is expressed in developing brain neurons and serves a cell survival-promoting function. J Mol Neurosci. 2000; 14: 3-15.

14. Fu W, Lu C, Mattson MP. Telomerase mediates the cell survivalpromoting actions of brain-derived neurotrophic factor and secreted amyloid precursor protein in developing hippocampal neurons. The Journal of Neuroscience. 2002; 22: 10710-10719.

15. Lu C, Fu W, Mattson MP. Telomerase protects developing neurons against DNA damage-induced cell death. Developmental Brain Research. 2001; 131: 167-171.

16. Zhu H, Fu W, Mattson MP. The Catalytic Subunit of Telomerase Protects Neurons Against Amyloid $\beta$-Peptide-Induced Apoptosis. Journal of Neurochemistry. 2000; 75: 117-124.

17. Klapper W, Shin T, Mattson MP. Differential regulation of telomerase activity and TERT expression during brain development in mice. Journal of Neuroscience Research. 2001; 64: 252-260.

18. Lee J, Jo YS, Sung YH, Hwang IK, Kim H, Kim SY, et al. Telomerase deficiency affects normal brain functions in mice. Neurochemical research. 2010; 35: 211-218.

19. Saretzki G. Telomerase beyond telomeres: new roles for an old enzyme, IN: Telomerase: Composition, Function and Clinical implications, pp 1-34, Nova Science Publishers. 2010; ISBN: 978-1-61668-957-5

20. Saretzki G. Extra-telomeric functions of human telomerase: cancer, mitochondria and oxidative stress. Current Pharmaceutical Design. 2014; 20: 6386-6403.

21. Santos JH, Meyer JN, Skorvaga M, Annab LA, Van Houten B. Mitochondrial hTERT exacerbates free-radical-mediated mtDNA damage. Aging Cell. 2004; 3: 399-411.

22. Ahmed S, Passos JF, Birket MJ, Beckmann T, Brings S, Peters H, et al. Telomerase does not counteract telomere shortening but protects mitochondrial function under oxidative stress. Journal of Cell Science. 2008; 121: 1046-1053.

23. Haendeler J, Dröse S, Büchner N, Jakob S, Altschmied J, Goy C, et al. Mitochondrial Telomerase Reverse Transcriptase Binds to and Protects Mitochondrial DNA and Function From Damage. Arteriosclerosis, Thrombosis, and Vascular Biology. 2009; 29: 929-935.

24. Singhapol C, Pal D, Czapiewski R, Porika M, Nelson G, Saretzki GC. Mitochondrial Telomerase Protects Cancer Cells from Nuclear DNA Damage and Apoptosis. PLoS ONE. 2013; 8: e52989.

25. Chung J, Khadka P, Chung IK . Nuclear import of hTERT requires a bipartite nuclear localization signal and Akt-mediated phosphorylation. J Cell Sci. 2012; 125 (Pt 11): 2684-97.

26. Seimiya H, Sawada H, Muramatsu Y, Shimizu M, Ohko K, Yamane K, et al. Involvement of 14-3-3 proteins in nuclear localization of telomerase. EMBO J. 2000; 19: 2652-61.

27. Haendeler J, Hoffmann J, Brandes RP, Zeiher AM, Dimmeler S. Hydrogen
Peroxide Triggers Nuclear Export of Telomerase Reverse Transcriptase via Src Kinase Family-Dependent Phosphorylation of Tyrosine 707. Molecular and Cellular Biology. 2003; 23: 4598-4610.

28.Indran IR, Hande MP, Pervaiz S. hTERT overexpression alleviates intracellular ROS production, improves mitochondrial function, and inhibits ROS-mediated apoptosis in cancer cells. Cancer Res. 2011; 71(1): 266-76.

29.Sharma NK, Reyes A, Green P, et al. Human telomerase acts as a hTRindependent reverse transcriptase in mitochondria. Nucleic Acids Res. 2012; 40: 712-25.

30.Simonicova L, Dudekova H, Ferenc J, Prochazkova K, Nebohacova M, Dusinsky R, et al. Saccharomyces cerevisiae as a model for the study of extranuclear functions of mammalian telomerase. Curr Genet. 2015; 61(4): 517-27.

31.Ghavami S, Shojaei S, Yeganeh B, Ande SR, Jangamreddy JR, Mehrpour $\mathrm{M}$, et al. Autophagy and apoptosis dysfunction in neurodegenerative disorders. Prog Neurobiol. 2014; 112: 24-49.

32. Kovalenko OA, Kaplunov J, Herbig U, Detoledo S, Azzam EI, Santos $\mathrm{JH}$, et al. Expression of (NES-) hTERT in cancer cells delays cell cycle progression and increases sensitivity to genotoxic stress. PLoS One. 2010; 5 (5): e10812.

33. Kovalenko OA, Caron MJ, Ulema P, Medrano C, Thomas AP, Kimura M, et al. A mutant telomerase defective in nuclear-cytoplasmic shuttling fails to immortalize cells and is associated with mitochondrial dysfunction. Aging Cell. 2010a; 9(2): 203-19.

34. Liu K, Hodes RJ, Weng N. Telomerase activation in human Tlymphocytes does not require increase in telomerase reverse transcriptase (hTERT) protein but is associated with hTERT phosphorylation and nuclear translocation. J Immunol. 2001; 166: 4826-4830.

35.Akiyama M, Hideshima T, Hayashi T, Tai YT, Mitsiades CS, Mitsiades N, et al. Nuclear Factor- $\mathrm{\kappa B}$ p65 mediates tumor necrosis factor a-induced nuclear translocation of telomerase reverse transcriptase protein. Cancer Res. 2003; 63 18-21.

36. Akiyama M, Yamada 0, Hideshima T, Yanagisawa T, Yokoi K, Fujisawa $\mathrm{K}$, et al. TNFalpha induces rapid activation and nuclear translocation of telomerase in human lymphocytes. Biochem Biophys Res Commun. 2004; 316: 528-32.

37.Iannilli F, Zalfa F, Gartner A, Bagni C, Dotti CG. Cytoplasmic TERT Associates to RNA Granules in Fully Mature Neurons: Role in the Translational Control of the Cell Cycle Inhibitor p15INK4B. PLoS ONE. 2013; 8: e66602.

38.Spilsbury, A, Miwa, S, Attems, J, Saretzki G The role of telomerase protein TERT in Alzheimer's disease and in tau-related pathology in vitro. J of Neuroscience. 2015; 35(4): 1659-74.

39. Mattson MP, Duan W, Chan SL, Cheng A, Haughey N, Gary DS, et al. Neuroprotective and neurorestorative signal transduction mechanisms in brain aging: modification by genes, diet and behavior. Neurobiol Aging. 2002; 23(5): 695-705.

40.Eitan E, Tichon A, Gazit A, Gitler D, Slavin S, Priel E. Novel telomeraseincreasing compound in mouse brain delays the onset of amyotrophic lateral sclerosis. EMBO Molecular Medicine. (2012a); 4: 313-329.

41. Kang HJ, Choi YS, Hong SB, Kim KW, Woo RS, Won SJ, et al. Ectopic expression of the catalytic subunit of telomerase protects against brain injury resulting from ischemia and NMDA-induced neurotoxicity. The Journal of Neuroscience: the official journal of the Society for Neuroscience. 2004; 24: 1280-1287.

42.Barnham KJ, Masters CL, Bush AI. Neurodegenerative diseases and oxidative stress. Nat Rev Drug Discov. 2004; 3(3): 205-14.

43.Chou JL, Shenoy DV, Thomas N, Choudhary PK, Laferla, Goodman FM, et al. Early dysregulation of the mitochondrial proteome in a mouse model of Alzheimer's disease. J. Proteomics. 2011; 74 (4): 466-479. 
44. Chiang YJ, Hemann MT, Hathcock KS, Tessarollo L, Feigenbaum L, Hahn WC, et al. Expression of telomerase RNA template, but not telomerase reverse transcriptase, is limiting for telomere length maintenance in vivo. Molecular and Cellular Biology. 2004; 24: 7024-7031.

45. Götz J, Chen F, van Dorpe J, Nitsch RM. Formation of Neurofibrillary Tangles in P301L Tau Transgenic Mice Induced by A $\beta 42$ Fibrils. Science. 2001; 293: 1491-1495.
46.Zhang B, Chen L, Swartz KR, Bruemmer D, Eum SY, Huang W, et al. Deficiency of telomerase activity aggravates the blood-brain barrier disruption and neuroinflammatory responses in a model of experimental stroke. J Neurosci Res. 2010; 88(13): 2859-68.

47.González-Giraldo Y, Forero DA, Echeverria V, Gonzalez J, ÁvilaRodriguez M, Garcia-Segura LM, et al. Neuroprotective effects of the catalytic subunit of telomerase: A potential therapeutic target in the central nervous system. Ageing Res Rev. 2016; 28: 37-45. 